\title{
Multiple Scedosporium apiospermum abscesses in a woman survivor of a tsunami in northeastern Japan: a case report
}

\author{
Yutaka Nakamura ${ }^{1 *}$, Yu Utsumi ${ }^{1}$, Naomi Suzuki ${ }^{1}$, Yoshio Nakajima ${ }^{1}$, Okinori Murata ${ }^{1}$, Nobuhito Sasaki ${ }^{1}$, \\ Hiroo Nitanai ${ }^{1}$, Hiromi Nagashima ${ }^{2}$, Shinya Miyamoto ${ }^{2}$, Jun Yaegashi ${ }^{3}$, Tomoki Hatakeyama ${ }^{3}$, Yoshihiro Shibano ${ }^{3}$, \\ Kyoko Yarita ${ }^{4}$, Katsuhiko Kamei ${ }^{4}$, Toshihide Nakadate ${ }^{5}$, Shigeatsu Endo ${ }^{5}$, Yasuo Terayama ${ }^{6}$ and Kohei Yamauchi ${ }^{1}$
}

\begin{abstract}
Introduction: Scedosporium apiospermum is increasingly recognized as a cause of localized and disseminated mycotic infections in near-drowning victims.

Case presentation: We report the case of a 59-year-old Japanese woman who was a survivor of a tsunami in northeastern Japan and who had lung and brain abscesses caused by S. apiospermum. Initially, an aspergillus infection was suspected, so she was treated with micafungin. However, computed tomography scans of her chest revealed lung abscesses, and magnetic resonance images demonstrated multiple abscesses in her brain. $S$. apiospermum was cultured from her bronchoalveolar lavage fluid, and antimycotic therapy with voriconazole was initiated. Since she developed an increase in the frequency of premature ventricular contractions, an adverse drug reaction to the voriconazole was suspected. She was started on a treatment of a combination of low-dose voriconazole and liposomal amphotericin B. After combination therapy, further computed tomography scans of the chest and magnetic resonance images of her brain showed a demarcation of abscesses.

Conclusions: Voriconazole appeared to have a successful record in treating scedosporiosis after a near drowning but, owing to several adverse effects, may possibly not be recommended. Thus, a combination treatment of lowdose voriconazole and liposomal amphotericin B may be a safe and effective treatment for an S. apiospermum infection. Even though a diagnosis of scedosporiosis may be difficult, a fast and correct etiological diagnosis could improve the patient's chance of recovery in any case.
\end{abstract}

\section{Introduction}

Tsunami lung occurs when a person who is overwhelmed by tsunami waves inhales saltwater contaminated by mud and microorganisms. Some microorganisms regarded as harmless saprophytes are, with increasing frequency, being reported to cause serious or lethal infections, even in immunocompetent individuals. Scedosporium apiospermum is increasingly recognized as a cause of localized and disseminated mycotic infections in near-drowning victims. This ubiquitous fungus is present in soil, manure, sewage, and

\footnotetext{
* Correspondence: ICB75097@nifty.com

'Division of Pulmonary Medicine, Allergy, and Rheumatology, Department of Internal Medicine, Iwate Medical University School of Medicine, 19-1

Uchimaru, Morioka, 0208505, Japan

Full list of author information is available at the end of the article
}

polluted waters. Here, we describe the case of a patient with both lung and brain abscesses caused by $S$. apiospermum and the therapeutic approach used for patients with tsunami lung associated with near drowning.

\section{Case presentation}

A previously healthy 59-year-old Japanese woman had been swept away by the tsunami that struck the Sanriku coast in northeastern Japan. She aspirated saltwater contaminated with soil and heavy oil, which had run out from the capsized ships. She swam back ashore and reached a shelter at a gymnasium by herself. She was transferred to a neighboring hospital, where she developed respiratory failure within a few hours of admission, was intubated, and was placed on a respirator. Management with positive end-expiratory pressure, fluid

\section{Ciomed Central}


restriction, and broad-spectrum antibiotics did not lead to any improvement in her pneumonia. She was referred to our hospital five days after she had almost drowned. Upon admission (on day six), she had a fever of $39^{\circ} \mathrm{C}$, a blood pressure of $142 / 92 \mathrm{~mm} \mathrm{Hg}$, and a tachycardia of 112 beats per minute. There was no evidence of trauma or fractures anywhere on her body. Auscultation of the chest revealed some crackles in both lungs. Her heart sounds were normal, and the results of an abdominal examination were normal. Her hemoglobin level was $10.0 \mathrm{~g} / \mathrm{dL}$, her platelet count was $27.4 \times 10^{10} / \mathrm{L}$, and her white cell count $12.9 \times 10^{9} / \mathrm{L}$, and she had a neutrophilia of $11.7 \times 10^{9} / \mathrm{L}$.

Her serum albumin was reduced to $23 \mathrm{~g} / \mathrm{L}$, and her alkaline phosphatase was increased to 357U/L (normal range of 99 to $340 \mathrm{U} / \mathrm{L})$. Her $\mathrm{C}$-reactive protein (CRP) was raised to $185 \mathrm{mg} / \mathrm{L}$. Serum urea, creatinine, electrolytes, glucose, and coagulation were within normal reference ranges. A urine analysis showed normal findings. A 12-lead electrocardiogram showed sinus tachycardia. Computed tomography (CT) scanning of her head was normal and that of her chest revealed infiltration and nodular lesions in both sides of her lungs (Figure 1). Blood cultures were taken from our patient after admission and showed no growth. A bronchoalveolar lavage (BAL) was performed by inserting a flexible fiberoptic bronchoscope. The BAL fluid contained oil, sand, and small pieces of wood. Tsunami lung was suspected, and, in addition to an antifungal treatment, broad-spectrum antibiotics were used, starting with $500 \mathrm{mg}$ of

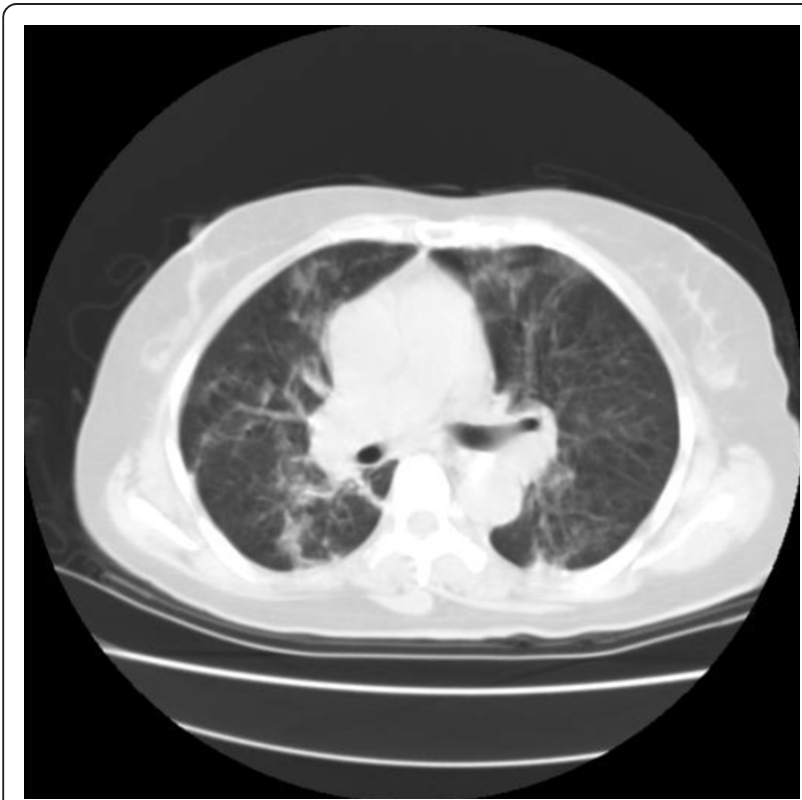

Figure 1 The chest computed tomography scan from admission shows that nodular shadows and filtrates are scattered in both lungs. carbapenem intravenously three times a day (t.i.d.), 500 mg of pazufloxacin intravenously twice a day (b.i.d.), $150 \mathrm{mg}$ of micafungin intravenously once a day (s.i.d.), and steroid pulse therapy. Nevertheless, a follow-up CT scan of the lung on day 28 showed multiple nodular lesions of the bilateral lung and a cavity in the upper left lobe (Figure 2$)$, and the level of $(1 \rightarrow 3)-\beta$-D-glucan (from 11.7 to $110 \mathrm{pg} / \mathrm{mL}$ ) had increased. Therefore, trimethoprim-sulfamethoxazole (trimethoprim $4.8 \mathrm{mg} / \mathrm{kg} \mathrm{t}$. i.d.) and $500 \mathrm{mg}$ of vancomycin intravenously four times a day were added to the treatment. At this time, a new BAL was examined, and although BAL fluid cultures for common and anaerobic bacteria were also negative, filamentous fungi, which seemed to be the cause in this case, were seen. On the basis of these results, the antimycotic therapy was switched to voriconazole (initially 6 $\mathrm{mg} / \mathrm{kg}$ b.i.d. followed by $4 \mathrm{mg} / \mathrm{kg}$ b.i.d.). On day 36 , our patient developed a palpitation, and the frequency of premature ventricular contractions (PVCs) increased, although electrolyte imbalance and high concentrations of voriconazole in the plasma were not examined. An adverse drug reaction of voriconazole was suspected, and the drug was withdrawn. Our patient was started on liposomal amphotericin B (2.5 mg/kg s.i.d.) along with trimethoprim-sulfamethoxazole, vancomycin, and $500 \mathrm{mg}$ of levofloxacin intravenously s.i.d. After the start of liposomal amphotericin B therapy, our patient's condition ameliorated and she was weaned successfully from mechanical ventilation. Magnetic resonance imaging of her head on day 63 revealed multiple brain

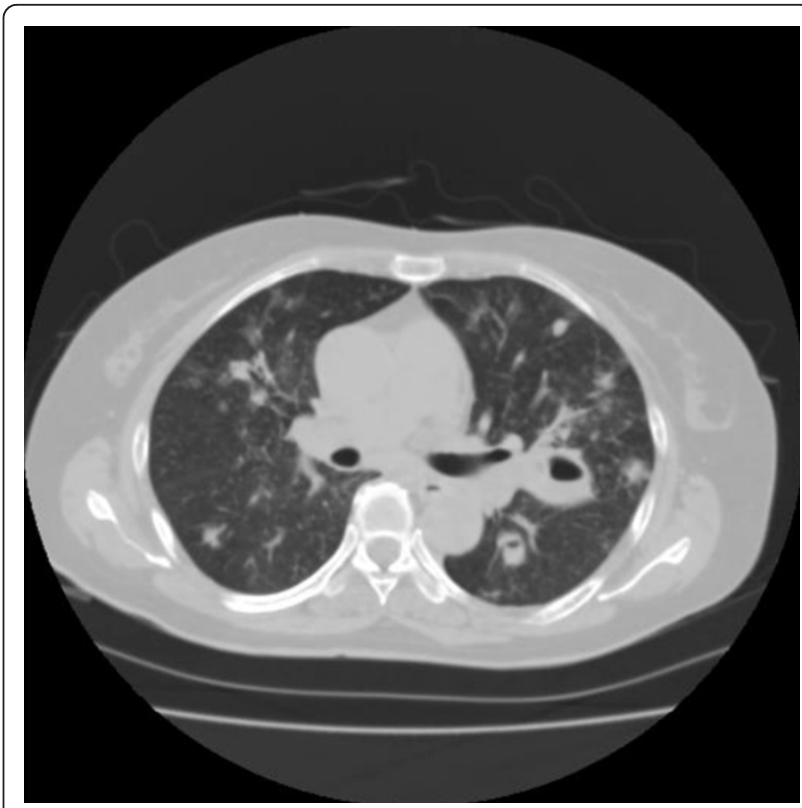

Figure 2 The chest computed tomography scan from day 28 shows a cavity in the left upper lobe, which has a thick wall and is surrounded by consolidation. 
abscesses (Figure 3a,b), but the results of her physical and neurological examinations were unremarkable, except for the presence of a mild fever. A lumbar puncture was performed but showed no white cells and no identifiable organisms on a Gram stain or upon a culture of the cerebrospinal fluid. On day 69, the diagnosis of $S$. apiospermum infection was confirmed by polymerase chain reaction and DNA sequencing of cultured filamentous fungi obtained from the previous BAL specimen. Since the isolate was considered susceptible to voriconazole (minimum inhibitory concentration [MIC] of $0.09 \mu \mathrm{g} / \mathrm{mL}$ ), our patient was started on lowdose intravenous voriconazole (initially $6 \mathrm{mg} / \mathrm{kg}$ b.i.d. followed by $2 \mathrm{mg} / \mathrm{kg}$ b.i.d.) with liposomal amphotericin B (MIC of $1 \mu \mathrm{g} / \mathrm{mL}$ ), which showed a relatively low MIC against $S$. apiospermum. After 15 days of treatment with a combination of voriconazole and liposomal amphotericin $\mathrm{B}$, further magnetic resonance imaging of the head showed a demarcation of abscesses in the brain on day 88 (Figure 3c,d). After the start of a combination therapy, the low-grade fever recurred, and the levels of both CRP and $(1 \rightarrow 3)-\beta$-D-glucan declined. On day 93, a chest CT showed the size of the cavity, the consolidation at the left upper lobe had reduced in size, and many of the nodular shadows in the lung fields had

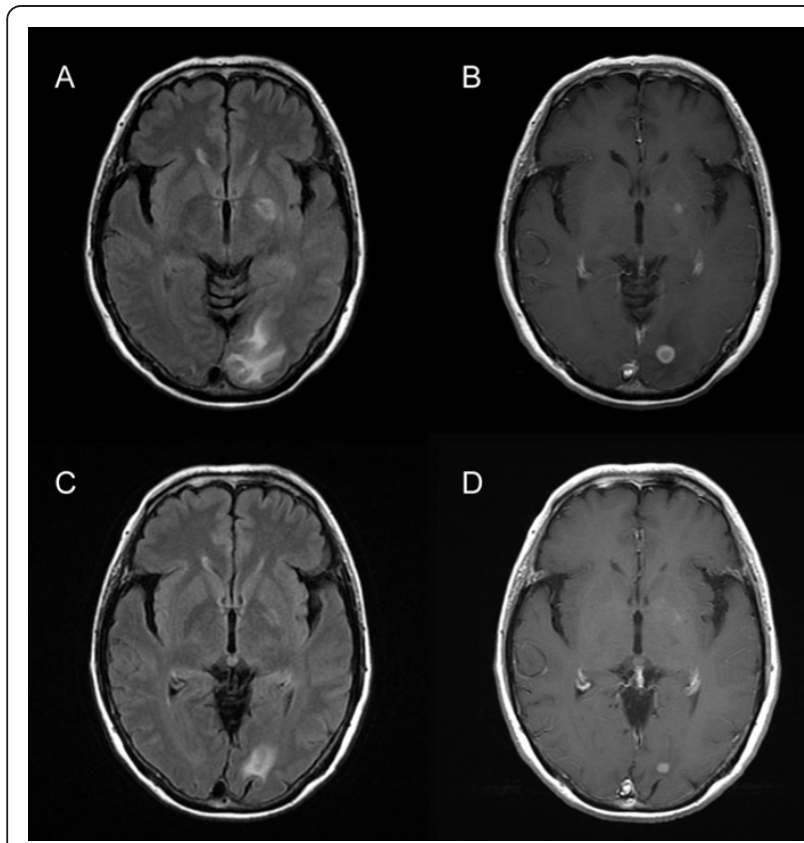

Figure 3 The axial fluid-attenuated inversion-recovery (FLAIR) image shows multiple hyperintensity lesions in the left occipital white matter and in the left internal capsule (a). The post-contrast T1-weighted image shows multiple rounded lesions with peripheral capsular enhancement in the corresponding area (b). Almost four weeks later, multiple hyperintensity lesions in the left occipital white matter and the left internal capsule (c) as well as multiple enhanced lesions (d) are diminished in size and intensity. disappeared (Figure 4). Oral administration of voriconazole was substituted with intravenous voriconazole and included the addition of oral levofloxacin $500 \mathrm{mg}$ and clarithromycin $400 \mathrm{mg}$. The voriconazole serum levels, checked regularly, were found to be within therapeutic limits. No recurrence was noted, and our patient was transferred to a hospital in her hometown 96 days after treatment had been initiated.

\section{Discussion}

S. apiospermum generally shows a high propensity to cause central nervous system (CNS) infections. The mode of $S$. apiospermum invasion and its subsequent expansion to the CNS remains ambiguous. Probable pathophysiological mechanisms of S. apiospermum infection after a near drowning include a local spread from sites near the brain, such as the paranasal sinuses [1] or cribiform plate [2], and a hematogenous spread from the lungs.

Voriconazole has shown efficacy in CNS-related scedosporiosis refractory to treatment with other antifungal agents, and several case reports and open-label clinical studies have provided in vivo evidence of the efficacy and tolerability of voriconazole as a method of therapy in patients with Scedosporium infections [3-5]. Another study describes voriconazole concentrations that are higher in cerebrospinal fluid than in plasma in a female patient [6]. Though generally well tolerated, voriconazole therapy may be associated with adverse events like transient visual disturbances, hepatotoxicity, skin rashes

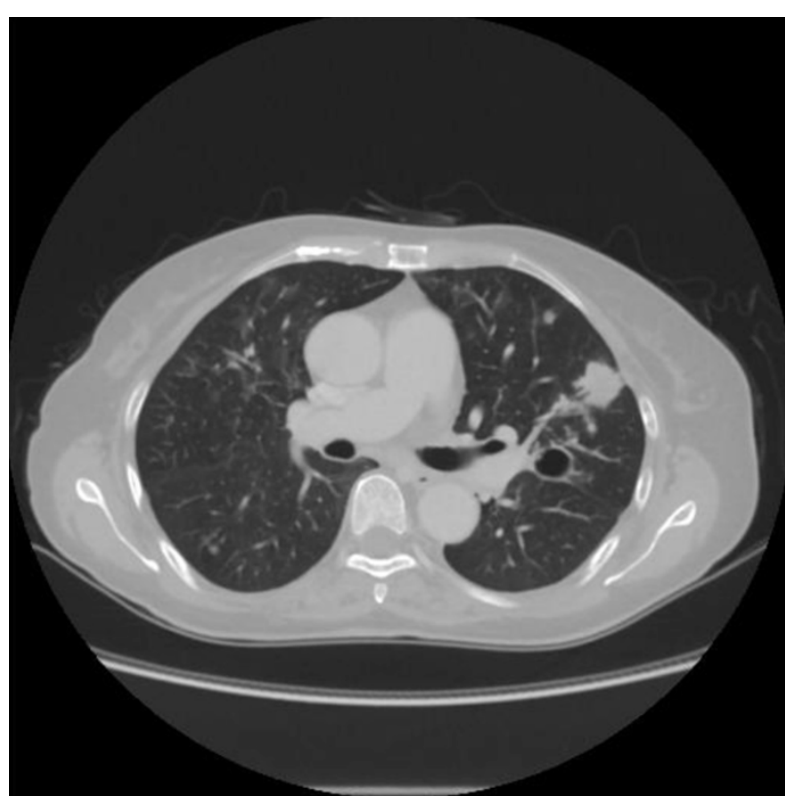

Figure 4 The chest computed tomography scan from day 93 shows the cavity at the left upper lobe, which has become reduced in size. 
$[7,8]$, and life-threatening ventricular arrhythmia [9]. In our patient, voriconazole appeared to be effective but was accompanied by PVCs and so was discontinued. However, in vitro susceptibility studies have shown that S. apiospermum isolates are susceptible to voriconazole and appear to have variable susceptibility to amphotericin B [5,10-13]. In our patient, susceptibility testing according to the methods of the Clinical and Laboratory Standards Institute was performed; the testing showed that the MIC for amphotericin B was relatively low and in agreement with the previous results that had higher MICs for flucytosine, fluconazole, itraconazole, miconazole, and micafungin. Because enhanced antifungal activity has been demonstrated in vitro for combinations of amphotericin B plus azoles [14,15], we had decided to add low-dose voriconazole to amphotericin B while using electrocardiographic monitoring. After voriconazole therapy was reinstated in a low dose, adverse events, such as PVCs, were not seen. The synergistic interaction on our patient can be evaluated even though low-dose voriconazole was administered.

\section{Conclusions}

In our patient, there was no evidence of fungal infections during the first month after her near-drowning experience. In addition, repeated blood cultures remained negative for fungi. Initially, aspergillosis was suspected, so our patient was treated with micafungin, although aspergillosis after near drowning seems to be rarer than scedosporiosis [12]. Another victim of the tsunami, who was also referred to our hospital, was a Japanese woman with Scedosporium prolificans. Before the fungus was diagnosed, she received treatments of antibiotics, steroids, and extracorporeal membrane oxygenation. She continued to deteriorate because of infection and succumbed to septic shock. In this fatal case, the lack of defined abscesses and specific symptoms of fungi infection prevented antifungal treatment from being administered. Because the detection of Scedosporium was confined to the BAL from the lung abscess, it was obviously too late for a successful antifungal therapy. Katragkou and colleagues [12] reported that the median 'time to diagnosis of Scedosporium infection' was 28 days. This could be attributed to the low sensitivity of routine culture methods. In our patient, since conidia had not formed, filamentous fungi were cultured repeatedly and, on day 64 , were submitted to polymerase chain reaction and DNA sequencing.

The tsunami wiped out much of the medical infrastructure, and many medications were not available. Therefore, we propose that voriconazole be used empirically and very early in patients who have nearly drowned. When voriconazole should not be used because of adverse effects, the use of combined therapies can be a promising clinical approach for combating infections caused by multidrug-resistant fungi. A fast and correct etiological diagnosis could improve the patient's outcome in any case.

\section{Consent}

Written informed consent was obtained from the patient for publication of this case report and any accompanying images. A copy of the written consent is available for review by the Editor-in-Chief of this journal.

\section{Abbreviations}

BAL: bronchoalveolar lavage; b.i.d.: twice a day; CNS: central nervous system; CRP: C-reactive protein; CT: computed tomography; MIC: minimum inhibitory concentration; PVC: premature ventricular contraction; s.i.d.: once a day; ti..d.: three times a day.

\section{Acknowledgements}

The authors would like to acknowledge Y Hatakeyama and M Shibanai (Iwate Medical University School of Medicine, Morioka, Japan) for their help in performing the laboratory work.

\section{Author details}

'Division of Pulmonary Medicine, Allergy, and Rheumatology, Department of Internal Medicine, Iwate Medical University School of Medicine, 19-1 Uchimaru, Morioka, 0208505, Japan. ${ }^{2}$ Department of Pulmonary Medicine, Iwate Prefectural Miyako Hospital, 1-11-26 Sakikuwagasaki, Miyako, 0270096, Japan. ${ }^{3}$ Department of Internal Medicine, Saiseikai Iwaizumi Hospital, 19-1 Nakaya, Iwaizumi aza, Iwaizumi town, Shimoheigun, 0270501, Japan. ${ }^{4}$ Medical Mycology Research Center, Chiba University, 1-8-1 Inohana, Chiba, 2608673, Japan. ${ }^{5}$ Department of Critical Care Medicine, Critical Care and Emergency Center, Iwate Medical University School of Medicine, 19-1 Uchimaru, Morioka, 0208505, Japan. ${ }^{6}$ Division of Neurology and Gerontology, Department of Internal Medicine, Iwate Medical University School of Medicine, 19-1 Uchimaru, Morioka, 0208505, Japan.

\section{Authors' contributions}

All of the authors were involved in the conception of the case report, the data collection, and literature review as well as in writing the manuscript. YN was a major contributor in writing the manuscript. YU and NS performed the bronchoscopy. KY and KK identified the filamentous fungi as $S$. apiospermum and S. prolificans. All authors read and approved the final manuscript.

\section{Competing interests}

The authors declare that they have no competing interests.

Received: 23 June 2011 Accepted: 25 October 2011

Published: 25 October 2011

\section{References}

1. Fisher JF, Shadomy S, Teabeaut JR, Woodward J, Michaels GE, Newman MA, White E, Cook P, Seagraves A, Yaghmai F, Rissing JP: Near-drowning complicated by brain abscess due to Petriellidium boydii. Arch Neurol 1982, 39:511-513.

2. Hachimi-Idrissi S, Willemsen M, Desprechins B, Naessens A, Goossens A, De Meirleir L, Ramet J: Pseudallescheria boydii and brain abscesses. Pediatr Infect Dis J 1990, 9:737-741.

3. Perfect JR, Marr KA, Walsh TJ, Greenberg RN, DuPont B, de la TorreCisneros J, Just-Nübling G, Schlamm HT, Lutsar I, Espinel-Ingroff A, Johnson E: Voriconazole treatment for less-common, emerging, or refractory fungal infections. Clin Infect Dis 2003, 36:1122-1131.

4. Schwartz S, Reisman A, Troke PF: The efficacy of voriconazole in the treatment of 192 fungal central nervous system infections: a retrospective analysis. Infection 2011, 39:201-210.

5. Espinel-Ingroff A, Johnson E, Hockey H, Troke P: Activities of voriconazole, itraconazole and amphotericin $B$ in vitro against 590 moulds from 323 
patients in the voriconazole phase III clinical studies. J Antimicrob Chemother 2008, 61:616-620.

6. Elter T, Sieniawski M, Gossmann A, Wickenhauser C, Schröder U, Seifert H, Kuchta J, Burhenne J, Riedel KD, Fätkenheuer G, Cornely OA: Voriconazole brain tissue levels in rhinocerebral aspergillosis in a successfully treated young woman. Int J Antimicrob Agents 2006, 28:262-265.

7. Lazarus HM, Blumer JL, Yanovich S, Schlamm H, Romero A: Safety and pharmacokinetics of oral voriconazole in patients at risk of fungal infection: a dose escalation study. J Clin Pharmacol 2002, 42:395-402.

8. Tan K, Brayshaw N, Tomaszewski K, Troke P, Wood N: Investigation of the potential relationships between plasma voriconazole concentrations and visual adverse events or liver function test abnormalities. J Clin Pharmacol 2006, 46:235-243.

9. Eiden C, Peyrière H, Tichit R, Cociglio M, Amedro P, Blayac JP, Margueritte G, Hillaire-Buys D: Inherited long QT syndrome revealed by antifungals drug-drug interaction. J Clin Pharm Ther 2007, 32:321-324.

10. Meletiadis J, Meis JF, Mouton JW, Rodriquez-Tudela JL, Donnelly JP, Verweij PE, EUROFUNG Network: In vitro activities of new and conventional antifungal agents against clinical Scedosporium isolates. Antimicrob Agents Chemother 2002, 46:62-68,

11. Cuenca-Estrella M, Gomez-Lopez A, Mellado E, Buitrago MJ, Monzon A, Rodriguez-Tudela JL: Head-to-head comparison of the activities of currently available antifungal agents against 3,378 Spanish clinical isolates of yeasts and filamentous fungi. Antimicrob Agents Chemother 2006, 50:917-921.

12. Katragkou A, Dotis J, Kotsiou M, Tamiolaki M, Roilides E: Scedosporium apiospermum infection after near-drowning. Mycoses 2007, 50:412-421.

13. Troke $\mathrm{P}$, Aguirrebengoa K, Arteaga C, Ellis D, Heath CH, Lutsar I, Rovira M, Nguyen Q, Slavin M, Chen SC, Global Scedosporium Study Group: Treatment of Scedosporiosis with voriconazole: clinical experience with 107 patients. Antimicrob Agents Chemother 2008, 52:1743-1750.

14. Walsh TJ, Peter J, McGough DA, Fothergill AW, Rinaldi MG, Pizzo PA: Activities of amphotericin $B$ and antifungal azoles alone and in combination against Pseudallescheria boydii. Antimicrob Agents Chemother 1995, 39:1361-1364.

15. Rodríguez MM, Calvo E, Serena C, Mariné M, Pastor FJ, Guarro J: Effects of double and triple combinations of antifungal drugs in a murine model of disseminated infection by Scedosporium prolificans. Antimicrob Agents Chemother 2009, 53:2153-2155.

doi:10.1186/1752-1947-5-526

Cite this article as: Nakamura et al:: Multiple Scedosporium apiospermum abscesses in a woman survivor of a tsunami in northeastern Japan: a case report. Journal of Medical Case Reports 2011 5:526.

\section{Submit your next manuscript to BioMed Central and take full advantage of:}

- Convenient online submission

- Thorough peer review

- No space constraints or color figure charges

- Immediate publication on acceptance

- Inclusion in PubMed, CAS, Scopus and Google Scholar

- Research which is freely available for redistribution

Submit your manuscript at www.biomedcentral.com/submit 